8

\title{
Manufacturing Systems Engineering for the Extended Enterprise
}

\author{
E. Corradi ${ }^{\dagger}$, A. Bartolotta ${ }^{\dagger}, M$. Garetti $^{\dagger}, M$. Rabe $^{\ddagger}$ and \\ A. Raimondo ${ }^{\S}$ \\ ${ }^{\dagger}$ Politecnico di Milano \\ piazza Leonardo Da Vinci 32, I-20133 Milano, Italy \\ ${ }^{\ddagger}$ IPK Fraunhofer Gesellschaft \\ Pascalstraße 8-9, D-80587Berlin, Germany \\ ${ }^{\S}$ Candy Elettrodomestici, \\ via privata E. Fumagalli, I-20047 Brugherio (MI), Italy
}

\begin{abstract}
This paper describes an ongoing research project, funded by the EC Esprit program, aiming at developing a software workbench supporting the manufacturing systems engineering process. The system provides a user-friendly and powerful environment in which tools supporting the conception, design and evaluation of manufacturing systems will be integrated in a common framework. The foundation of the workbench is a descriptive method of the domain of manufacturing systems (based on the object-oriented methodology), enabling the description of all the aspects of a generic manufacturing system. The paper describes the main design issues of the workbench and presents its logical architecture; then it underlines how this system can allow the coordinated work of many design teams of a distributed company thanks to the standard descriptive method used to describe manufacturing systems.

It is possible to foresee that such a system could have a dramatic impact on the manufacturing system engineering process in the extended enterprise environment.
\end{abstract}

\section{Keywords}

Manufacturing systems engineering, object-oriented modeling 


\section{MANUFACTURING SYSTEM ENGINEERING}

Manufacturing Systems Engineering (MSE) is a recently recognized multidisciplinary engineering function covering all traditional forms of engineering concerned with production: it ranges from design of manufacturing systems, to logistics design, to flow control philosophy selection, to production organization. Recently the need for frequent restructuring of manufacturing systems, for new faster product-to-market cycles and for planning facilities of the extended enterprise is calling for MSE process optimization. Surprisingly in this important area a comprehensive information technology (IT) support is still missing (Garetti and Bartolotta, 1995). In this context, an EC project is ongoing to study, develop and prototypically test a software system enabling the management, on a distributed environment, of the manufacturing systems design process.

\section{MSE IN THE EXTENDED ENTEPRISE}

The diffusion of the concept of extended enterprise greatly enhances the importance of a well supported process in the manufacturing systems engineering area. Globalization is bringing about the integration and coordination of the internationally distributed facilities and capabilities of a single company into a coherent global production resource, in order to achieve powerful competitive advantages in the process. This trend towards integration pushes many manufacturing companies with distributed plants to define a standardized and integrated approach to the manufacturing system engineering process for all the plants of the company. In fact the planning of remote facilities and the updating of their structure strongly require a sound integration among the design processes at the various sites, to get optimizations coming from the integrated view of the whole system.

Today the availability of information technology and telecommunication products supports distributed working and enables coordination and collaboration among several design teams located in different geographical sites, regardless of their physical location.

It is evident that this widened approach to manufacturing system design firstly requires a deep analysis and reengineering of the MSE process, in order to establish the design issues of an integrated workbench able to support the approach.

\section{STATE OF THE ART OF THE MSE PROCESS}

While in the product design area $\mathrm{CAD} / \mathrm{CAM} / \mathrm{CAE}$ technologies provide a powerful and comprehensive support to the design process, and in the production management area a lot of IT tools are supporting the production planning process, a lack does exist in the MSE area where a single approach is not available yet, 
offering a complete framework (a 'platform') to handle the brain intensive work of designing a modern manufacturing system: so, at a large extent, the MSE process continues to be left to the intuitive judgement of the production managers' mind.

In the meantime, production decentralization and internationalization of enterprises, involving remote plants and distributed engineering offices, all stress the importance of a standardized and integrated approach to the MSE process. Last but not least, the absence of a MSE platform means the lack of a method to store suitably the manufacturing system architecture, entailing a missing link between product design and production management processes (while these processes structurally share a great amount of data with the MSE process).

\section{DESIGN ISSUES OF THE MSE WORKBENCH}

\subsection{Modelling and re-engineering of the MSE process}

The objective to enhance the production engineer's work productivity can be achieved through the use of a suitable computer-aided workbench able to provide a support in the MSE process.

This workbench must provide all the functionalities needed during the design process and support distributed working, which typically characterizes of the design activities. Therefore it represents an integration environment, where all the tools supporting the design of manufacturing systems are integrated in a common framework; of course, all the functionalities and information of the system must be accessed and managed by the design teams distributed across a company.

According to these principles, an analysis of the entire MSE process has been performed according to a model-based approach: referring to an industrial casestudy (Rabe et al., 1997), subprocesses were identified and each subprocess was modeled in terms of input-output specifications (Mertins and Jochem, 1992). The analysis gave the opportunity to identify the most important process weaknesses and to define a re-engineered MSE process which can be effectively supported by a computer-aided workbench: these requirements lead to the definition of the functionalities, tools, data structures and information needed during the reengineered design process.

\subsection{Formal representation of manufacturing systems}

In order to effectively support the designer in the MSE process and to provide all the required functionalities, the software workbench assists in the construction of different models of the manufacturing system. In fact, within the workbench environment, the manufacturing systems design process is seen as carried out in some steps, where several models of the manufacturing system are generated and evaluated and a satisfying solution is identified through an iterative process. 
The descriptive method we propose allows to define a structured representation of manufacturing systems (that we called manufacturing entity structure, MES), which is a structure of objects, where the entities (building blocks), their attributes and the relationships among the entities are defined, thus providing a standardized data format for the description of manufacturing systems.

According to the descriptive method, the representation of manufacturing systems can be structured into three different aspects:

- The structural aspect: it contains the structural (static) definition of the system, including workers, production facilities (including tools, jigs and fixtures), material handling equipment and other supplementary devices.

- The technological aspect: it defines the transformational (functional) view of the system, considering the conversion process of the factors of production.

- The management aspect defines the operating procedures of production, constituting the so-called management cycle, i.e. planning, implementation and control.

Each aspect only captures some features of the manufacturing system reality, whereas the whole system is exhaustively described putting the three aspects together. The manufacturing entity structure contains the building blocks for the modelling of all the three aspects explained above.

As regards the implementation of the descriptive method, the object-oriented approach presents characteristics that make it particularly suitable to meet the requirements of the manufacturing entity structure (Bartolotta and Garetti, 1996).

This descriptive method also provides the semantic unification necessary for the sharing of information among the different tools/models that have to be integrated.

Around this 'basic stone', all other elements follow:

- the integration platform linking together already existing MSE tools;

- the new organizational model of the MSE process;

- the integration link between product design and production management.

\subsection{The workbench logical architecture}

In Figure 1, the logical architecture of a workbench implementing the descriptive method is reported. The kernel of the system, named CAMSE (Computer-Aided Manufacturing Systems Engineering), contains a series of logical modules corresponding to the functionalities provided by the system. Accordingly, the logical architecture should be seen as a set of tools the designer will freely use in his activity. The system also includes interfaces to external systems (in particular CAD and simulation). Let's illustrate this picture considering the involved data structures first and the logical modules performing the various functions afterwards. 


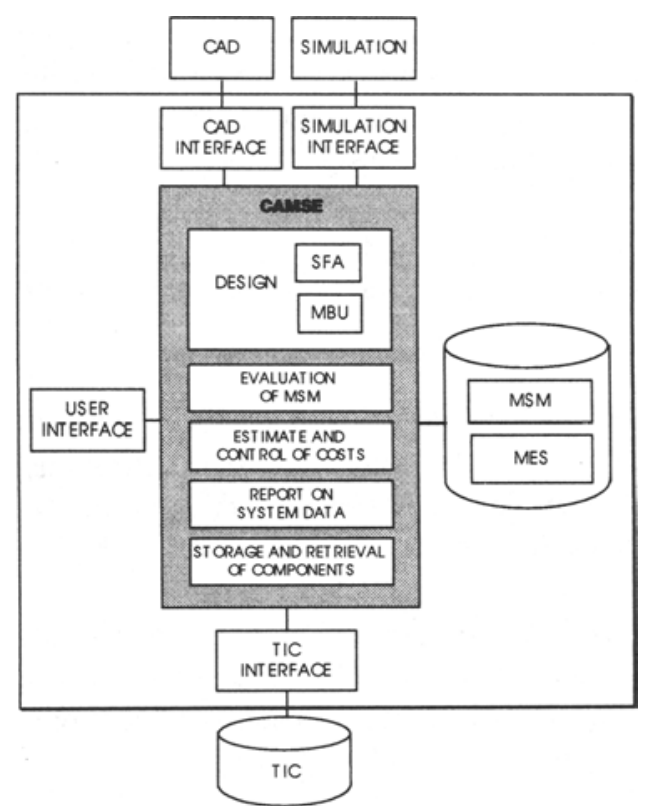

Figure 1 The system logical architecture.

\section{Data structures}

Two different data structures have been depicted in the logical architecture: the system database, internal, and the technical information catalogue, external to the system.

The system database is composed of two parts: the persistent part of the database stores the manufacturing entity structure (MES), i.e. all the building blocks (object classes) which are used to build the different models of any manufacturing system, while the temporary part of the database contains the models, called manufacturing system models (MSM), built or stored the design process instancing the MES classes.

The manufacturing system model is the object model of the manufacturing system, of which two different views are foreseen: i) the system logical view (SLV), representing the logical view of the manufacturing system model in terms of logical objects and relationships among them (described selecting and linking symbolic icons representing machines, handling systems and other plant elements from a 'catalogue' of objects); ii) the rough layout view (RLV) representing the physical layout of the manufacturing system, obtained embedding the logical view with further data related to the physical dimension of the plant components, and visualizing the manufacturing system to scale, as in a sort of CAD system. 
The Technical Information Catalogue is an archive of information on manufacturing systems, subsystems and components stored in different formats, such as an electronic format, or as a pointer to traditional archives (folders, floppy disks, etc.), provided as a consulting support for designers.

\section{Logical modules}

The following is the list of main modules obtained identifying the needs of MSE.

\section{A. Design}

Within the design module, the following modules are established:

- a system flow analysis (SFA) module, that, starting from data on products to be manufactured in the production system (production mix, bills of material and process plans), allows the generation of the model of the production flow within the manufacturing system;

- a model building and updating (MBU) module, which supports the designer in the construction of the manufacturing system models, providing him with an user-friendly interface for selecting and linking objects from a catalogue realized through an object-oriented database.

\section{B. Storage and retrieval of Components}

This module allows to store and retrieve information about manufacturing systems, subsystems, single plant components. Besides it must provide the designer with the possibility to enrich the content of the database using industry-specific libraries of plant components.

\section{Evaluation of Manufacturing systems Models}

This module allows both the interfacing of the system to commercial off-the-shelf systems, like queue theory or simulation tools, and contains sub-modules (tools) able to evaluate the performances of the manufacturing system models.

\section{Estimate and control of plant costs}

The module allows to estimate the costs of the designed production system.

\section{E. Report on System Data}

The module makes information available in output, as tables, graphs and figures, so to be used by management systems, such as procurement, detailed design, etc. 


\section{THE ROLE OF THE WORKBENCH IN THE EXTENDED ENTERPRISE}

The efficiency and potentiality of the software workbench can be used to support and manage the MSE process of extended enterprises. This can be achieved exploiting the standardization action performed by the descriptive method used in the workbench enabling the coordinate work of many design teams located in different sites.

\subsection{IT technologies supporting the Extended Enterprise}

The managing of the MSE process for the extended enterprise is made possible by the availability of computer networking and telecommunication technologies, which enable to synthesize geographically dispersed knowledge, information and expertise. As known, Web technologies allow the visibility and distribution of information and services at a low cost and on any platform. The Web provides a non proprietary environment, which presents no constraints on the type of data managed and on the modes of insertion, modification and consultation of data. Furthermore, it allows to manage extended data types: multimedia data, from alphanumeric to formatted texts and images, HTML files, audio and video, VRML files.

It is therefore possible to organize Web sites supporting multimedia data, like text, sounds, graphics, etc., which can be easily updated, and are quite inexpensive to maintain. Other services provided by the net such as electronic mail, fax, internet phone, file transfer through FTP, videoconferencing, etc. are also useful for supporting cooperative activities and teamwork together with specific groupware applications which are also available in the Web.

In conclusion the WWW technologies offer services and information at a low cost, in an open environment, multimedia and independent of the platform and thus represent an interesting technological solution to manage Extended Enterprises.

\subsection{The performances of the 'extended' workbench}

The integration of the software workbench within the Web, whether INTERNET, or a company network (INTRANET), gives the possibility to access to all the information and services available in the network, makes it easier to communicate and share data among different design teams using the workbench and promotes also the cooperation with external partners, such as suppliers of plant components or third parties designers.

It is evident that the activities of design of a manufacturing system can be greatly speeded up and improved if an easy retrieval and access to data used during the 
process and an effective exchange of information among the design teams is provided.

As widely described in the previous section, the workbench includes two databases storing all the information and data structures used during the design process: the system database and the technical information catalog.

The temporary part of the system database (MSM) allows to store models of manufacturing systems related to former and ongoing design projects. Therefore, if the workbench is integrated within the Web, the databases of the various workbenches can be seen as a distributed database containing the archive of the updated configurations of all the existing plants of a company, located in different geographical sites; the archive, accessible by all the design teams through the network, represents a standardization and integration element within the company. Besides, plant configurations adopted in different plants, which are stored in the database, can be easily consulted during the design activity and properly re-used in models of new plants under construction, thus improving the development time and the quality of the design.

The technical information catalogue (TIC) contains data on plant components, coming from different sources, which are typically consulted during the design activity. Therefore the integration of this database into the Web enables to manage this kind of data in a non proprietary environment, providing an easy access to them. It is also possible to organize Web sites containing all these generic information: for instance, sites including electronic catalogues, constantly updated, of all the plant components produced by a certain supplier, including text and non text material, sounds, graphics, videoclips, etc.

The MSE process can be greatly improved by an efficient exchange of information among the engineering teams involved in the design activities. To this aim, the integration of the workbench within the Web allows the design teams to communicate and exchange data and information by using the services and the tools available in the network supporting information flow, such as electronic mail, internet phone, file transfer through FTP, videoconferencing, etc. These tools allow interaction among remote engineering teams and also collaboration with external partners, such as suppliers of plant components.

The design process can be supported also by the use of specific tools managing cooperative activities and supporting teamwork, available in the network. These tools allow to have a shared management of information on ongoing projects: designers can consult information on the current project, check its advancement status, organize working groups on parts of the project.

\section{EXPECTED BENEFITS}

The benefits expected from the use of the software workbench in the extended enterprise environment are: 
- to reduce the effort of creating and evaluating the model of a manufacturing system: a large library of reusable plant components and subsystems could be created, where a manufacturing company could store also the plant configurations adopted in different plants; then the model of a new manufacturing system will be developed by properly selecting previously stored objects from the library, thus reducing the development time and improving the quality of design;

- to enforce standardization in manufacturing system design: the common descriptive method of the software workbench will be the same for all the design teams of a company. Therefore it represents a standardization element, urging the designers to choose standard components during the project development;

- to enhance coherence and coordination among different engineering teams, whether they will be dislocated in different geographical sites (multi-site design), or they will be working, in a design site, on modules of the same manufacturing system: in fact, referring to a common (even if distributed) database including the updated configurations of all the existing plants of a company geographically distributed described with the same standard method, every design team will have easy access to projects developed by other teams; the database thus represents an integration element, allowing to have a general view of all the plants and the alignment of information. Besides coordination of work among different design teams can be further enhanced by the use of specific communication tools which are available in the network;

- to reduce the time spent in re-design and reconfiguration of manufacturing systems: once made the one-time strain of creating the initial base models of all manufacturing systems of a company, the incremental effort to make a change in a current plant, such as the introduction of a new machine, will be slight, thank to the possibility to utilize and update the model previously stored;

- to minimize the retro-fitting and fine tuning associated with implementation of a manufacturing system: the possibility to carry out a fast computeraided analysis and evaluation of the designer's idea will entail presumably a better-quality solution;

- to reduce inconsistency and redundancy of manufacturing system data: during the development of the manufacturing system engineering process, the same common base representation will be employed, after properly translation, by the different modeling tools; this representation, starting from a conceptual configuration, will be progressively detailed as the design process evolves. 


\section{REFERENCES}

Bartolotta, A. and Garetti, M. (1996) Object-oriented representation of manufacturing systems: trends and perspectives. Proceedings of the Advances in Production Management Systems conference, IFIP, Kyoto

Garetti M. and Bartolotta A. (1995) General concepts of a Manufacturing Systems Engineering workbench as a tool for the reengineering of manufacturing systems. In Browne J. and O'Sullivan D. (eds.) Re-engineering the enterprise, IFIP, Chapman \& Hall

Mertins K. and Jochem R. (1992) An object-oriented method for Integrated Enterprise Modelling as a basis for Enterprise coordination, International Conference on Enterprise Integration Modeling Technology (ICEIMT), Hilton Head (South Carolina), US Air Force-Integration Technology Division

Rabe, M.; Müller, W.; Gronchi, D. and Garetti, M.: Modelling the Manufacturing System Engineering Process. Il progetto comunitario PLANTFABER, Mailand 1997.

Anna Bartolotta is a PhD student of the Dipartimento di Economia e Produzione of the Politecnico di Milano. She received the degree in Management Engineering in 1994 at the Politecnico di Milano presenting a degree thesis entitled 'Framework for a Manufacturing System Engineering Workbench'. Her research interests include advanced tools and techniques for the design and management of manufacturing systems.

Elena Corradi holds a master degree in Management Engineering from the Politecnico di Milano. At present she is in charge of a study grant from Univerlecco. Her fields of research are the design of production systems and the relationships between product and process design.

Marco Garetti is Professor of Industrial Technology at the Dipartimento di Economia e Produzione of the Politecnico di Milano, Italy. After graduating in Mechanical Engineering in 1971 at Politecnico, he began his career at Alfa Romeo. In 1974 he joined Politecnico di Milano, working in the field of industrial production, automated production systems and CIM. Prof. Garetti is a senior member of the Computer and Automated Systems Association of the Society of Mechanical Engineering (CASA/SME), a member of IFIP W.G. 5.7, a member of the editorial board of PIXEL (an Italian review on CAD-CAM) and of the International Journal of Production Planning and Control.

$\mathrm{He}$ is active in organising seminars and post-graduate education courses on production management and automated manufacturing. His actual fields of interest 
are Design of Production Systems, Production Management, Expert Systems in Manufacturing, Simulation and Computer Integrated Manufacturing. $\mathrm{He}$ is at present in charge of the Diploma Universitario in Logistics and Production Engineering of the Politecnico di Milano.

Markus Rabe studied physics at the University of Konstanz. He has been working since 1986 at the Fraunhofer Institute for Production Systems and Design Technology (IPK Berlin) within the systems planning division, where he is at present head of the production planning department. His activities are on factory planning and enterprise modelling, with focus on simulation. He is member of ASIM and head of the BerlinSimulation Demonstration Center.

Adriano Raimondo graduated in Mechanical Engineering and spent six years as Responsible of Production at 'F.lli Borletti' (Milan), three years as Responsible of Manufacturing Engineering at 'Borletti F. B.' (S. G. su Legnano) and two years as Responsible of the project for the restructuring and re-organisation of a plant at 'B\&B' (Como). Singe 1991 he is responsible of Time \& Methods and efficiency at the Candy Group.

(*) Plantfaber is the name of the EC project 'Plantfaber: An integrated software workbench as a tool for the re-engineering of the Manufacturing System Engineering Process', co-sponsored by the European Commission, IV Framework Programme - DG III Industry Information Technology - Technical Domain 7: Technology for Business Processes (Task 7.6: Business Methodologies and Tools). The partners of the project are two industrial users (Candy Elettrodomestici (I) and Convey Satafim (E)), two research institutes (MIP of Politecnico di Milano (I) and IPK Fraunhofer Institute of Berlin (D)) and two software developers (LIPRO from Berlin (D) and Spaziosystem (I), who is the main contractor of the project). The Plantfaber project started on May 1996 and will finish on October 1998. 